\title{
The Spin-Up Rate of the White Dwarf in GK Per
}

\author{
Christopher W. Mauche \\ Lawrence Livermore National Laboratory, \\ L-473, 7000 East Avenue, Livermore, CA 94550
}

\begin{abstract}
We use hard X-ray light curves measured by the Chandra HETG and RXTE PCA during the late rise and plateau phases of the 2002 March-April outburst of the intermediate polar GK Per to determine that its X-ray pulse period $P=351.332 \pm 0.002 \mathrm{~s}$. Combined with previous X-ray and optical measurements of the spin period of the white dwarf, we find that its spin-up rate $\dot{P}=0.00027 \pm 0.00005 \mathrm{~s} \mathrm{yr}^{-1}$.
\end{abstract}

\section{Introduction}

GK Per is an extraordinary cataclysmic variable: the first nova of the last century (Nova Per 1901), it contains a magnetic white dwarf, a large truncated accretion disk, and an evolved secondary; it is surrounded by a nova shell visible in the radio, optical, and X-ray wavebands; and it suffers large-amplitude $(\Delta V \approx 3)$, long $(\delta t \approx 60$ days $)$, but infrequent $(\Delta t \approx 900$ day) dwarf nova outbursts (Šmon 2002). On the strength of proposals submitted to the $X M M$ and Chandra Guest Observer programs, we were granted target-of-opportunity $X M M$ and Chandra (C. Mauche, PI) and RXTE (K. Mukai, PI) observations of GK Per during its 2002 March-April outburst with the goals of monitoring the X-ray light curve, pulse profile, and spectrum and obtaining the first grating spectra of GK Per to determine the nature of its complex X-ray spectrum (Şen \& Osborne 1998; Ezuka \& Ishida 1999). The number and dates of the observations were constrained by the small and decreasing angle between GK Per and the Sun, but we were fortunate to obtain $R X T E$ monitoring observations every few days throughout the outburst, an XMM observation on March 9 during the early rise to maximum, and Chandra HETG observations on March 27 and April 9 during the late rise and peak of the outburst. The XMM MOS and Chandra HETG confirm that the Fe $6.4 \mathrm{keV}$ fluorescent emission line is the strongest of the three Fe K-shell emission features, and the XMM RGS and Chandra HETG spectrometers reveal emission lines of $\mathrm{H}$-like $\mathrm{C}$; $\mathrm{H}$ - and $\mathrm{He}$-like $\mathrm{N}, \mathrm{O}, \mathrm{Ne}, \mathrm{Mg}, \mathrm{Si}$, and S; and possibly He-like Al (see Mukai et al. 2003 for a plot of the Chandra HETG spectrum). At least two sources of X-ray emission are implied by (1) the strong absorption of the continuum (which diminishes to zero for $\lambda \gtrsim 6 \AA$ ), (2) the presence of emission lines down to $\lambda \approx 30 \AA$, and (3) the fact that the short-wavelength $(\lambda=1-6 \AA)$ flux is modulated at the spin period of the white dwarf, while the long-wavelength $(\lambda=6-26 \AA)$ flux is steady. 


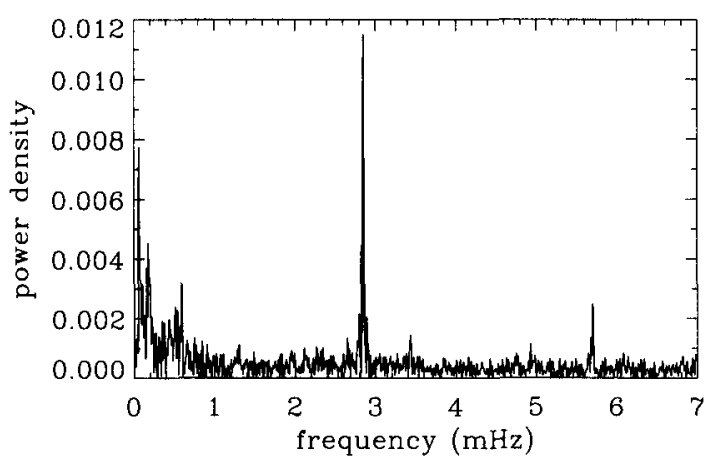

Figure 1. Power spectrum of the Chandra HETG first-order 1-6 $\AA$ light curve of GK Per. The peaks at $\nu=2.85$ and $5.68 \mathrm{mHz}$ are identified with the fundamental and first harmonic of the white dwarf spin period $P=351$.

\section{Spin Period of the White Dwarf}

To determine the spin period of the white dwarf of GK Per, we used data from the two $32 \mathrm{ks}$ Chandra observations separated by 13.2 days. Specifically, we created a count rate light curve of the Chandra HETG first-order $\lambda=1-6 \AA$ event data with $351.3 / 5=70 \mathrm{~s}$ bins, subtracted the mean of the two observations, and padded the interval between the two observations with zeros. The power spectrum of the resulting light curve is shown in Figure 1, with peaks at $\nu=5.68$, $2.85,0.59$, and $0.18 \mathrm{mHz}$, corresponding to periods $P=176,351,1700$, and 5520 $\mathrm{s}$; the $351 \mathrm{~s}$ period is the spin period of the white dwarf $P$, the $176 \mathrm{~s}$ period is its first harmonic $P / 2$, and the 5520 s period is the quasi-periodic oscillation discussed by Morales-Rueda, Still \& Roche (1999). Based solely on the power spectrum, the spin period of the white dwarf can be constrained only to lie in the range $P=349-353 \mathrm{~s}$, but by phasing the two Chandra HETG light curves, the number of cycles between the two observations is constrained to be $3250 \pm$ a few, corresponding to periods $P=351.332 \mathrm{~s} \pm$ multiples of $0.105 \mathrm{~s}$. This ambiguity is resolved by the light curves of the two intervening $R X T E$ PCA observations, which phase consistently with the two Chandra HETG light curves only for $P=351.332 \pm 0.002$ s (see Fig. 2).

\section{Spin-Up Rate of the White Dwarf}

The pulse period of GK Per has been measured previously in X-rays by Einstein (Eracleous, Patterson \& Halpern 1991) and EXOSAT (Watson, King \& Osborne 1985; Norton, Watson \& King 1988) and in the optical by Patterson (1991), who provides an excellent discussion of the measurements and uncertainties of the various determinations of this quantity. Patterson finds that the 1980 Einstein data are consistent with pulse periods $P=351.3423 \mathrm{~s} \pm$ multiples of $0.002 \mathrm{~s}$, the 1983-85 EXOSAT data are consistent with pulse periods $P=351.3372$, 351.3409 , or $351.3446( \pm 0.0007) \mathrm{s}$, and the 1989-91 optical data are consistent 

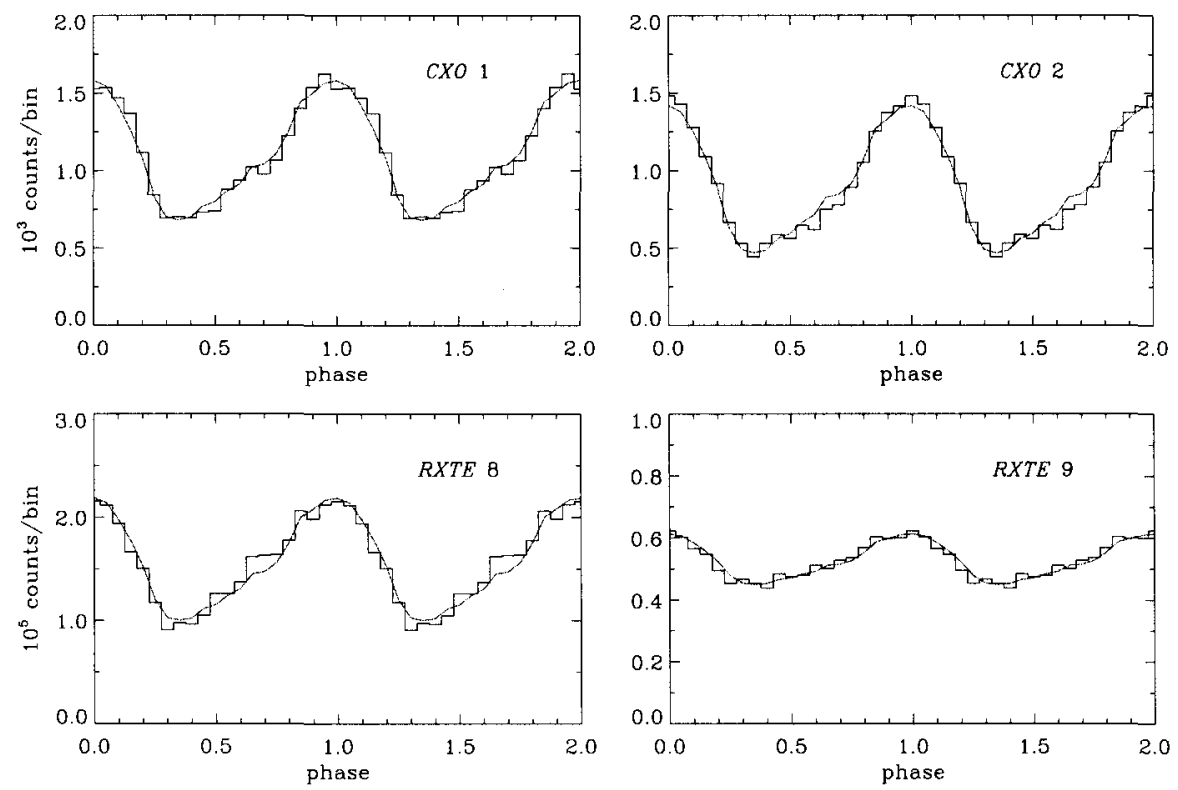

Figure 2. Phase-folded X-ray light curves of the first and second Chandra HETG and the intervening eighth and ninth RXTE PCA observations of GK Per. Data are shown by the histograms and the scaled mean phase-folded light curve is shown by the gray curves. Phase zero corresponds to HEJD 2452360.9113.

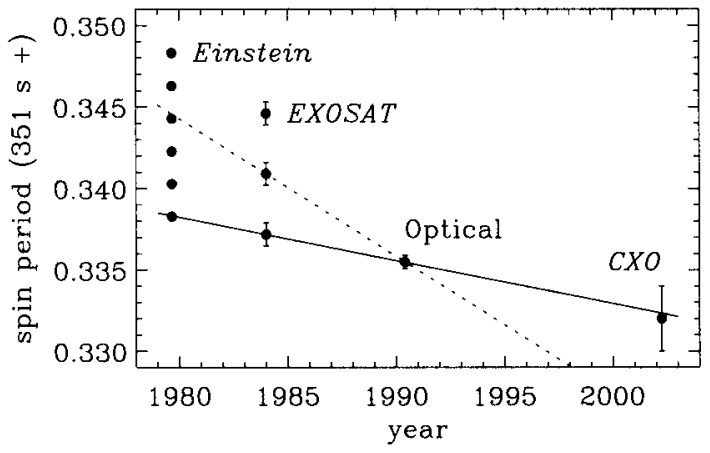

Figure 3. Spin period history of GK Per. The solid line is the spinup rate $\dot{P}=0.00027 \mathrm{~s} \mathrm{yr}^{-1}$ consistent with the Einstein, EXOSAT, optical, and Chandra and RXTE data, and the dotted line is the value $P=0.00084 \mathrm{~s} \mathrm{yr}^{-1}$ proposed by Patterson (1991). 
with a pulse period $P=351.3355 \pm 0.0004 \mathrm{~s}$; these data are plotted in Fig. 3 . Assuming that the pulse period at the epoch of the EXOSAT observations was 351.3409 s, Patterson proposed a spin-up rate $\dot{P}=0.0008 \mathrm{~s} \mathrm{yr}^{-1}$ (the dotted line in Fig. 3) for the white dwarf of GK Per, but cautioned that the result should be regarded as tentative, as there is an uncertainty in the cycle count. As shown in Figure 3, our determination of the pulse period of GK Per in 2002 is not consistent with a linear extrapolation of the spin-up rate proposed by Patterson, but is consistent with a spin-up rate $\dot{P}=0.00027 \pm 0.00005 \mathrm{~s} \mathrm{yr}^{-1}$ (the solid line in Fig. 3).

\section{Spin Ephemeris of the White Dwarf}

Given the results from the previous sections, the spin ephemeris of the white dwarf of GK Per is fully determined by specifying the date of some feature in the light curve. Following Watson, King \& Osborne (1985), we define phase zero to occur at pulse maximum, which, guided by the power spectrum, we determined by fitting a function $a+b \sin \left[2 \pi\left(\phi-\phi_{1}\right)\right]+c \sin \left[4 \pi\left(\phi-\phi_{2}\right)\right]$ to the mean phase-folded light curve. By this method, we find that X-ray pulse maximum of GK Per follows the ephemeris $T_{\max }=$ HEJD 2452360.9113 $( \pm 0.0001)+$ $0.00406634( \pm 0.00000002) E-(3.5 \pm 0.6) \times 10^{-14} E^{2}$. This ephemeris includes only the second secure determination of the spin period of the white dwarf of GK Per and the first secure determination of its spin-up rate.

Acknowledgments. This work benefited from discussions with J. Patterson, K. Mukai, and A. Norton. We thank J. Swank and H. Tananbaum for the grants of Director's Discretionary Time which made the RXTE and Chandra observations possible. Support for this work was provided in part by NASA through Chandra Award Number DD2-3014X issued by the Chandra X-Ray Observatory Center, which is operated by the Smithsonian Astrophysical Observatory for and on behalf of NASA under contract NAS8-39073. This work was performed under the auspices of the U.S. Department of Energy by University of California Lawrence Livermore National Laboratory under contract No. W-7405-Eng-48.

\section{References}

Eracleous, M., Patterson, J., \& Halpern, J. 1991, ApJ, 370, 330

Ezuka, H., \& Ishida, M. 1999, ApJS, 120, 277

Morales-Rueda, L., Still, M.D., \& Roche, P. 1999, MNRAS, 306, 753

Mukai, K., Kinkhabwala, A., Peterson, J.R., Kahn, S.M., \& Paerelset, F. 2003, ApJ, 586, L77

Norton, A.J., Watson, M.G., \& King, A.R. 1988, MNRAS, 231, 783

Patterson, J. 1991, PASP, 103, 1149

Şen, G., \& Osborne, J.P. 1998, in Wild Stars in the Old West, ed. S. Howell, E. Kuulkers, \& C. Woodward (San Francisco: ASP), 463

Šimon, V. 2002, A\&A, 382, 910

Watson, M.G., King, A.R., \& Osborne, J. 1985, MNRAS, 212, 917 